\title{
Performance économique des exploitations de bovin viande de la région du grand Cheliff (Algérie)
}

Sadoud, $M$.

Université Hassiba Benbouali de Chlef. Faculté des Sciences. Hay Es-salem. Chlef. Algérie.

\section{MOTS CLÉ SUPPLÉMENTAIRES}

Viande bovine.

Élevage.

Alimentation.

Marge.

Coût.

\section{RÉSUMÉ}

Dans le but de déterminer la performance économique ( $D A=$ Dinar Algerien; 1 Euro= 100 DA en 2011 ) des exploitations d'élevage bovin viande, une étude a été menée en 2011 dans la région du grand Cheliff au nord de l'Algérie. Un total de 102 exploitations a été enquêté, dont trois classes ont été identifiées. Les exploitations de la classe A présentent le coût le plus faible $(482,39 \mathrm{DA} / \mathrm{kg})$, suivies par celles des deux autres groupes $C$ et $B$ avec respectivement $(581,31$ DA et 585,58 DA). La recette unitaire de vente d'un bovin varie de $600,70 \mathrm{DA} / \mathrm{kg}$ vif pour les exploitations de la classe A à $601,17 \mathrm{DA} / \mathrm{kg}$ vif pour celles du groupe B. La recette moyenne unitaire de vente est de l'ordre de 600,93 DA/ kg.

Les exploitations de la classe $A$ affiche le prix de revient le plus faible, soit $465 \mathrm{DA} / \mathrm{kg}$, suivi par les exploitations de la classe B avec $563 \mathrm{DA} / \mathrm{kg}$, puis les exploitations de classe C avec un prix de 572,92 DA/kg. La marge brute au cours de l'année 2011 par tête bovine est de 78241,16 DA/tête. Elle varie d'un minimum de 60706 DA/tête pour les exploitations appartenant à la classe $C$ et d'un maximum de $91486 \mathrm{DA}$ /tête pour les exploitations du groupe B. Les exploitations de la Classe A peuvent être considérées comme le groupe le plus performant de l'ensemble des types distingués avec un coût de production parmi les plus bas. Par contre les autres groupes (Classe B et C) paraissent dans des conditions difficiles suite aux difficultés et contraintes des facteurs de production locaux ou importés.

\section{Economic performance of beef farms in the grand Cheliff region, Algeria}

SUMMARY

In order to evaluate the economic (DA= Algerian Dinar; 1 Euro= 100 DA in 2011) performance of beef farms, this study, carried out on a sample of 102 farms in the grand Cheliff (Algeria) during 2011 , resulted in the distinction of 3 classes $(A, B, C)$ of farms. Class $A$ farms showed the lowest production costs (482.39 DA) compared to class $B$ and $C$ pointing respectively 581.31 DA and 585.58 DA. The average unit selling price of live bovine was $600.93 \mathrm{DA} / \mathrm{kg}$. The lowest cost price $(465 \mathrm{DA} / \mathrm{kg})$ was presented by Class $\mathrm{A}$ farms, whereas the highest $(572.92 \mathrm{DA} / \mathrm{kg})$ was shown by Class $C$ farms. The average gross profit per head of livestock over the year 2011 was 78241.16 DA, ranging between a minimum of 60706 DA/head (Class $C$ farms) and a maximum of 91486 DA/head (Class B farms). As a result, Class $A$ farms was considered the best performing class as it showed the lowest production costs, compared to class $B$ farms $C$ under onerous conditions, following the various difficulties and constraints of either local or imported production factors.

INFORMACIÓN

Cronología del artículo.

Recibido/Received: 6.3.2015

Aceptado/Accepted: 28.10.2015

On-line: 10.12 .2015

Correspondencia a los autores/Contact e-mail:

m_sadoud@yahoo.fr; m.sadoud@univ-chlef.dz

\section{INTRODUCTION}

En Algérie, les productions animales sont de plus en plus diversifiées mais leurs performances sont encore faibles. En conséquence, la consommation de protéines animales reste modeste pour la majeure partie de la population: environ 16,5 g/habitant/jour alors que la norme recommandée par la FAO est fixée à $35 \mathrm{~g} /$ habitant/jour (Berchiche, 2003).

Le développement de l'élevage bovin a toujours constitué une priorité pour l'Algérie pour répondre aux besoins de la population en protéines animales (UBIFRANCE, 2014). Particulièrement pour les régions du nord du pays, qui sont considérées comme étant de grands consommateurs des viandes rouges (Sadoud, 2011). Mais cet élevage connait des contraintes techniques, économiques et sociales qui entravent son développement et se manifestent surtout par la forte dépendance (des importations d'aliments du bétail), une maitrise et une intégration faibles des modèles techniques adoptés se traduisant par une faiblesse des productivités (Abbas et al., 2011). 
Le présent travail a pour objectif de contribuer à une meilleure connaissance des performances économiques des exploitations d'élevage bovin viande dans les conditions de la région du périmètre irriguée du grand Cheliff.

\section{MATÉRIEL ET MÉTHODES}

Pour tenter de caractériser les exploitations agricoles pratiquant l'élevage bovin. Nous avons élaboré un questionnaire comportant deux parties:

Une première partie portant sur les caractéristiques générales des exploitations (taille, répartition et occupation des terres, localisation, production végétale, charge animale, main d'œuvre, cheptel).

Une seconde partie portant sur les caractéristiques de l'élevage bovin (effectif, reproduction, base fourragère, main d'œuvre, mécanisation, population bovine élevée).

Ces questionnaires nous permettent de collecter des informations qui vont servir à déterminer plusieurs indices ou critères afin de présenter les exploitations de façon succincte et d'établir des relations entre les différents indices et entre les aspects principaux lier à la production de viande.

Nous avons choisi des indicateurs socioéconomiques pour mesurer la performance des élevages enquêtés et qui sont: UGB totales (unité gros bétail), UGB bovines, l'importance des aliments achetés de l'extérieur. Nous avons également retenu des paramètres zootechniques liés à la reproduction et aux conduites alimentaires et sanitaires des vaches laitières (l'âge au premier vêlage, l'intervalle vêlage-vêlage, insémination/fécondation). Ainsi, nous avons retenu des paramètres économiques (coût de production, produit, prix de revient et la marge brute). L'ensemble de ces indicateurs (structurels et fonctionnels) choisis nous ont permis de distinguer une diversité des élevages et de comportement des éleveurs.

L'échantillon se compose de 102 exploitations et d'exploitants situés dans les plaines du haut et moyen Cheliff, plus connus sous la dénomination périmètre irrigué du haut Cheliff et moyen Cheliff.

Une version d'évaluation de Xlstat a été utilisée pour effectuer l'analyse statistique. Ce traitement statistique multi varié s'est avéré nécessaire en vue de différencier, clarifier et ordonner les types d'élevage face à la forte hétérogénéité constatée des exploitations bovines existantes.

\section{APPROCHE MÉTHODOLOGIQUE}

Le coût de production de l'atelier bovin viande est une méthode d'analyse de la performance de l'atelier bovin viande (Chambr'Agri, 2011).

Dans la détermination du coût on s'est servi des concepts suivants:

- Les charges opérationnelles ou proportionnelles affectées au système de production de vian de bovine et qui sont retenues dans le calcul du prix de revient concernant l'achat des aliments concentrés, le coût de production de fourrages, les frais d'élevage (les frais vétérinaires), et les autres charges opérationnelles.

- Les charges de structure: Elles comprennent les charges du matériel et les charges de main d'œuvre (Institut de l'élevage, 2011).

Le calcul du produit total de l'élevage comprend les recettes de la vente d'un bovin viande selon l'âge à la vente de l'animal et la croissance du gain moyen quotidien pour la race améliorée qui varie de 850 à 900 grammes/jour, que nous avons obtenu un bovin selon le poids vif à la vente (Géal et al. cité par Ali-Benamara, 1985), se sont généralement des veaux de boucheries (taurillons (légers, moyens et lourds) (Soltner, 1981) qui sont issus de troupeaux de races modernes qui sont la Pie-Rouge (Montbéliarde) et la Pie-Noir qui donnent naissance à des veaux qui ont un poids variant entre 35 et $40 \mathrm{~kg}$. La variation de la valeur des effectifs n'a pas été prise en considération dans l'évaluation du produit total de l'élevage, en raison de l'absence de données précises concernant le mouvement des animaux entre le début et la fin de la campagne agricole et en particulier celles relatives au poids des animaux concernant les mâles et les femelles de réforme. La méthode simplifiée de calcul du prix de revient d'un kg de viande consiste à affecter directement aux unités techniques de production (hectare ou unités techniques de production animale) ou de répartir directement entre ces unités techniques la totalité des charges de production.

Ainsi, le prix de revient est calculé en déduisant du coût de production les aides (Pons et Pouch, 2013).

La rentabilité d'un produit c'est l'aptitude à apporter de l'argent (Raimbault, 2004). Ainsi, la rentabilité est exprimée par le calcul des marges, ces dernières sont déterminées à l'aide de deux éléments : les charges et les produits.

La marge brute de la production de viande bovine est la différence entre le produit de l'activité qui est la vente du bovin de viande et les charges opérationnelles qui lui sont attribuées.

\section{Marge brute à la production= \\ Produit brut-Charge variable}

L'auto-approvisionnement en paille et foin est considéré comme l'équivalent d'un achat de ce produit au prix du marché. Les prix utilisés pour les autres aliments, notamment concentrés, sont des prix moyens relevés auprès des éleveurs au cours du déroulement de l'enquête.

Sur la base des indicateurs retenus, nous avons pu établir une différenciation des élevages de bovin viande et dresser une typologie. La construction d'une typologie est une démarche progressive, susceptible d'être remise en cause ou plus souvent complétée (Landais, 1987). 
Tableau I. Analyse des composantes principales (Principal component analysis).

\begin{tabular}{lrrrr}
\hline & \multicolumn{1}{c}{ F1 } & \multicolumn{1}{c}{ F2 } & \multicolumn{1}{c}{ F3 } & \multicolumn{1}{c}{ F4 } \\
\hline Valeur propre & 2,3976 & 1,7215 & 1,3952 & 1,0724 \\
Variabilité (\%) & 23,9761 & 17,2146 & 13,9520 & 10,7240 \\
Accumulé (\%) & 23,9761 & 41,1908 & 55,1428 & 65,8668
\end{tabular}

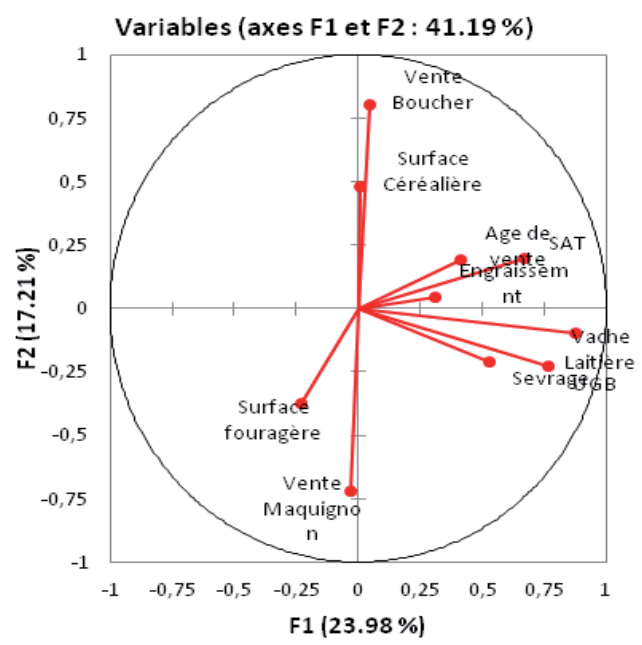

Figure 1. Cercle de corrélation des variables de l'ACP (Axe 1 et Axe 2) (Correlation circle of variables ACP (Axis 1 and Axis 2)).

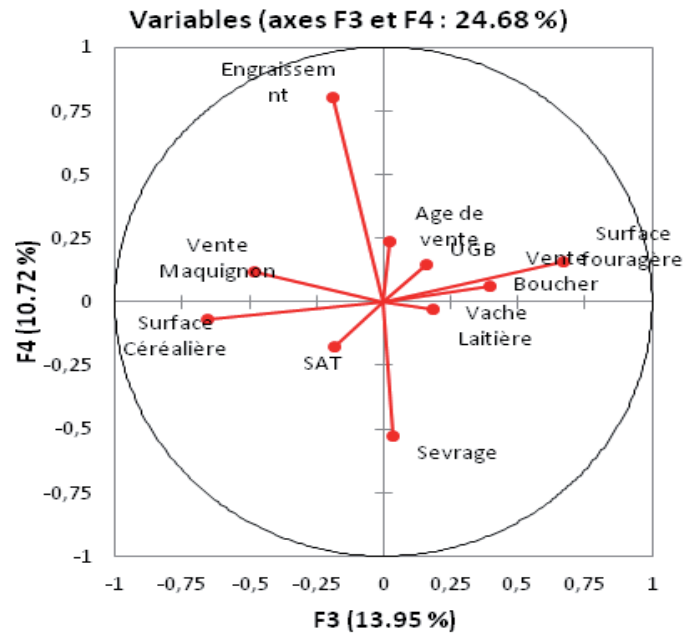

Figure 2. Cercle de corrélation des variables de 1'ACP (Axe 3 et Axe 4) (Correlation circle of variables ACP (Axis 3 and Axis 4)).

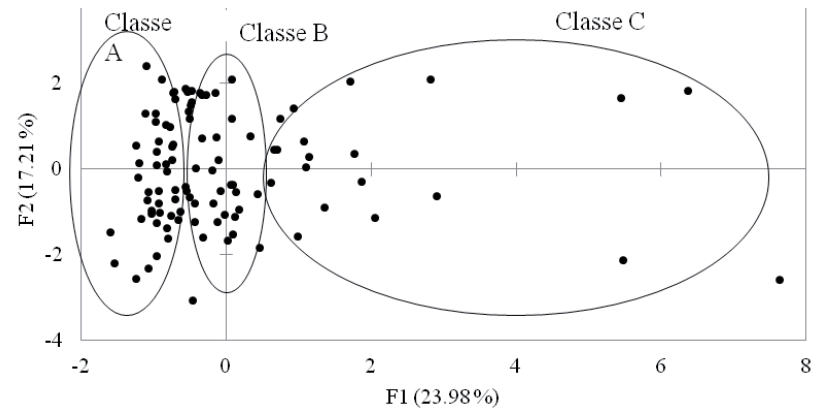

Figure 3. Schéma de la carte d'individus (Diagram of individuals map).

\section{RÉSULTATS}

\section{ANALYSE EN COMPOSANTES PRINCIPALES}

La variance totale expliquée par les quatre premiers axes était de $65,87 \%$ (tableau I), tandis que les autres axes avec des valeurs propres inférieures à 1 ont été exclus de l'analyse. Selon les cosinus carrés cinq variables (vaches laitières, unité gros de bétail, surface agricole totale, et vente de bovin au boucher) qui étaient bien représentées dans le plan $1 \times 2$.

Le premier axe avec $23,98 \%$ de la variance expliquée, était principalement lié positivement aux vaches laitières, unité gros bétail et superficie agricole totale, ce qui signifie que les fermes avec une grande superficie agricole ont été caractérisées par un grand nombre de vaches laitières et un effectif de cheptel bovin élevé (figures 1, 2 et 3 ).

Le deuxième axe $(17,21 \%)$ a été positivement liée aux éleveurs avec une grande superficie céréalière et vente de bovins plus souvent aux bouchers, alors que cet axe a été négativement liée aux éleveurs ayant consacrés une grande superficie pour la production de fourrage avec des ventes de bovins à des maquignons.

Trois variables (la surface fourragère et céréalière, la période d'engraissement et de sevrage) ont été bien représentés dans le plan $3 \times 4$. Le troisième axe $(13,95 \%$ de la variance expliquée) a été positivement lié à la superficie fourragère et négativement lié à la superficie céréalière. Enfin, le quatrième axe a été très positivement lié à la période d'engraissement et négativement au sevrage précoce ce qui signifie que les éleveurs pratiquant l'engraissement pratiquent un sevrage précoce.

\section{TYPOLOGIE DES EXPLOITATIONS BOVINES DE VIANDE}

L'analyse typologique des données collectées au cours de l'enquête nous permet d'identifier trois classes d'exploitations bovines de viandes, plus ou moins homogènes:

Classe A: Il s'agit des petits éleveurs à base de lait et de viande qui possèdent un effectif de vaches laitières et une superficie inférieure à 10VL (vache laitière) et 10 ha. La moyenne d'UGB bovin entre les exploitations de cette classe est de l'ordre de 3,18 UGB (unité de gros bétail). Donc ce sont des micro exploitations polyvalentes. Les exploitations de cette classe se caractérisent par une SAU moyenne de l'ordre de 10 ha, dont $65 \%$ sont réservés aux cultures fourragères. Ils ont un taux d'autonomie alimentaire moyen de $50 \%$, le reste de l'alimentation est acheté de l'extérieur et constitué principalement de concentré. La majorité d'entre eux assurent la vente des animaux mâles à l'âge de 18 à 24 mois après un engraissement de 2 à 3 mois, alors que pour le reste des exploitations la durée d'engraissement est de 6 mois. C'est un type qui assure la production d'une catégorie de mâles d'un poids vif moyen variant entre 400 et $450 \mathrm{~kg}$.

Classe B: Il s'agit des producteurs moyens, dont la superficie varie entre 10 et 30 ha. Le nombre des vaches laitières est inférieur à 10 vaches. La moyenne des UGB bovin entre les exploitations de cette classe est de 10 UGB. La part réservée aux cultures fourragères atteint 
en moyenne $35 \%$ de la SAU. Ils engraissent les animaux nés à l'exploitation, des mâles (veaux, taurillons et bœufs) et des femelles (vaches de réforme). La durée d'engraissement des taurillons est de 4 à 6 mois; les ventes s'effectueront à l'âge de 18 à 24 mois et même parfois à 30 mois.

Classe C: Il s'agit de grands éleveurs mixtes laitsviandes. Les exploitations sont de grande taille et la SAU est supérieure à 30 ha, l'effectif moyen des vaches laitières étant de 15 vaches. La moyenne d'UGB bovin entre les exploitations de cette classe est de l'ordre de 22,24 UGB. La part réservée aux cultures fourragères est de l'ordre de $41 \%$ de la SAU. La durée d'engraissement des mâles est de 6 à 9 mois et l'âge à la vente est d'environ 18 à 24 mois.

\section{LA PERFORMANCE DE L'ACTIVITÉ D'ÉLEVAGE BOVIN VIANDE}

La performance est un résultat optimal obtenu par l'utilisation la plus efficiente possible des ressources mises en œuvre.

L'appréhension de la performance de l'activité d'élevage bovin viande consistera à réaliser une analyse des coûts de production inhérents aux différentes phases de la production de viande bovine.

\section{ANALYSE DES COÛTS DE PRODUCTION}

Dans la mesure des charges alimentaires, il s'agit des fourrages consommés sur place, mesure directe à partir des données recueillies auprès des éleveurs.

L'auto-approvisionnement en paille et foin est considéré comme l'équivalent d'un achat de ce produit au prix du marché. Les prix utilisés pour les autres aliments, notamment concentrés, sont des prix moyens relevés auprès des éleveurs au cours du déroulement de l'enquête.

\section{Produits affectés à la viande:}

Ce poste se compose du produit principal qui est la viande et du sous produit de la viande qui est le fumier produit du troupeau. Le prix de revient du $\mathrm{kg}$ de viande est alors exprimé en tenant compte de la répartition des charges et des produits du système de production de bovin viande, par le rapport entre le total des charges affectées au bovin viande et le rendement moyen de l'animal.

L'analyse de la structure des charges relatives aux différentes classes d'élevage en termes de charges opérationnelles et de charges de structure révèle la prédominance des charges opérationnelles et en particulier des charges alimentaires pour l'ensemble des classes retenues mais avec des taux différents dans le total des charges.

\section{APPROCHE DU COÛT DE PRODUCTION}

La structuration du coût de production, toutes classes confondues, fait ressortir trois groupes de postes de coûts:

- Un poste dominant qui représente une moyenne de $73 \%$ des charges globales qui sont les charges alimentaires;

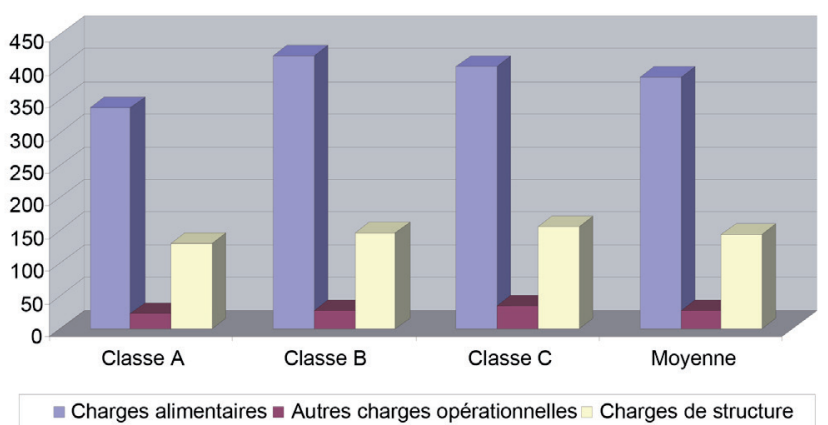

Figure 4. Structure des coûts de production par classe d'élevage en DA/kg vif (DA: Dinar Algérien; 1 Euro= 100 DA en 2011) (Structure of farm production costs per class in AD/kg bright (Algerian Dinar; 1 Euro= 100 DA in 2011)).

- un poste d'importance intermédiaire qui représente environs $13 \%$ des charges globales et qui se compose des charges liées au matériel et à la main d'œuvre;

- un poste d'importance faible représenté par les frais d'élevages (frais vétérinaires) et autres frais relatifs aux charges restantes (frais d'électricité, frais de pierre à lécher, frais de la paille pour litière) avec respectivement 3 et $1 \%$ des charges globales.

Notons que les charges consenties pour l'alimentation, la main d'œuvre et le matériel constituent environ $94 \%$ des charges affectées au bovin de viande. Tandis que le reste représente $6 \%$.

Les charges variables constituent ainsi une part de $70 \%$ du total du coût de production contre 30\% de charges de structure. Les charges moyennes annuelles par tête sont de l'ordre de 199673 DA. En conséquence, le coût de production moyen par $\mathrm{kg}$ de viande est estimé à 549,76 DA (figure 4).

Globalement, pour l'année 2011, les exploitations de la classe A présentent le coût le plus faible (482,39 DA), suivies par celles des deux autres groupes $C$ et $B$ avec respectivement (581,31 DA et 585,58 DA).

L'alimentation constitue le poste le plus important; la moyenne entre les trois classes est de l'ordre de $70 \%$ du coût total, elle varie entre $337,67 \mathrm{DA} / \mathrm{kg}$ de viande pour les exploitations de la classe A et 417,45 $\mathrm{DA} / \mathrm{kg}$ de viande pour celles de la classe B. Les autres charges opérationnelles représentées par les frais vétérinaires, d'électricité, de litière et de la pierre à lécher demeurent faibles et ne dépassent pas les 5\% du total des charges. Quant aux charges de structure, leur taux moyen représente $26,34 \%$ du coût global, soit $144,58 \mathrm{DA} / \mathrm{kg}$, variant entre un minimum de 130,19 DA/kg pour les exploitations considérées comme des petits producteurs d'animaux de viande du groupe A et $156,89 \mathrm{DA} / \mathrm{kg}$ de viande pour les exploitations de grande taille productrices mixte lait-viande du groupe C (figure 4).

\section{LES CHARGES OPÉRATIONNELLES}

Les charges opérationnelles moyennes par exploitation type s'élève à 189673 DA par tête, soit en moyenne $442,45 \mathrm{DA} / \mathrm{kg}$ vif de viande produite par tête. Ainsi, ces charges varient entre 402,23 DA pour les exploita- 


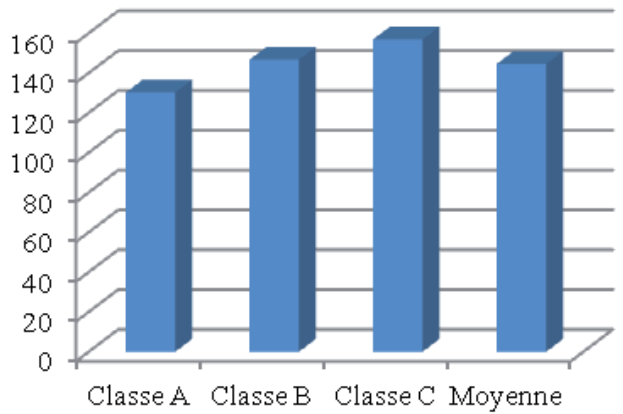

Figure 5. Répartition des charges de structure par classe d'élevage en $\mathrm{DA} / \mathrm{kg}$ vif (DA= dinar algérien; 1 Euro= 100 DA en 2011) (Allocation of overhead costs by farming class in $\mathrm{DA} / \mathrm{kg}$ bright (DA= Algerian dinar; 1 Euro= $100 \mathrm{DA}$ in 2011)).

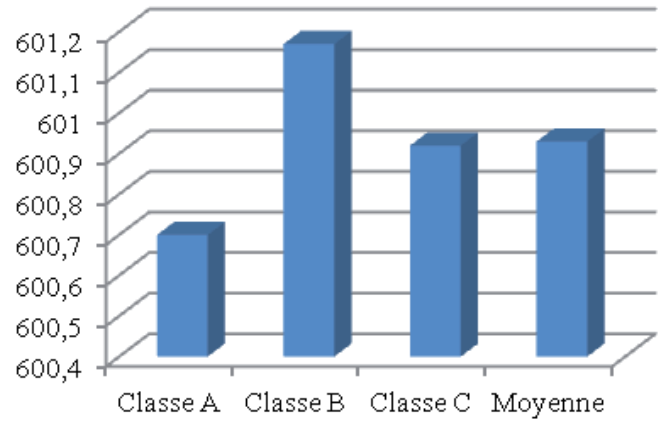

Figure 6. Structure des produits par classe d'élevage en $\mathrm{DA} / \mathrm{kg}$ vif $(\mathrm{DA}=$ dinar algérien; 1 Euro $=100 \mathrm{DA}$ en 2011) (Structure produced by breeding class $D A / k g$ bright (DA= Algerian dinar; 1 Euro $=100$ DA in 2011)).

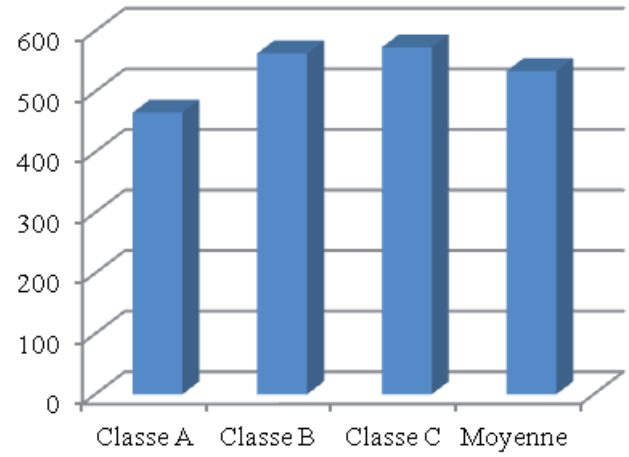

Figure 7. Structure du prix de revient par classe d'élevage en $\mathrm{DA} / \mathrm{kg}$ vif (DA= dinar algérien; 1 Euro= 100 DA en 2011) (Structure of livestock by class cost in DA/kg bright (DA= Algerian dinar; 1 Euro= 100 DA in 2011)).

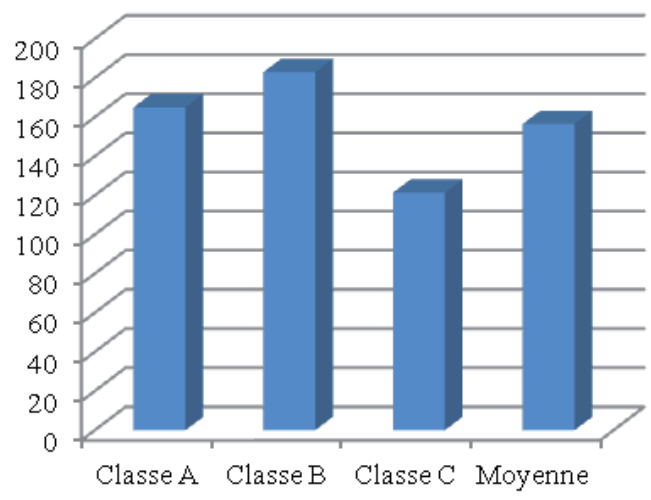

Figure 8. Structure de la marge brute par classe d'élevage en $\mathrm{DA} / \mathrm{kg}$ vif $(\mathrm{DA}=$ dinar algérien; 1 Euro= $100 \mathrm{DA}$ en 2011) (Structure of the gross margin per livestock class DA kg bright (DA= Algerian dinar; 1 Euro= $100 \mathrm{DA}$ in 2011)). tions appartenant à la classe A et $481,07 \mathrm{DA} / \mathrm{kg}$ pour les exploitations représentant la classe $\mathrm{B}$, soit respectivement $83,38 \%$ et $82,15 \%$ du coût total de production.

L'alimentation absorbe en moyenne $94 \%$ des charges opérationnelles. La rentabilité de l'élevage dépend de la manière dont est géré le poste alimentation. Les autres charges opérationnelles (frais vétérinaires, pierre à lécher, frais de la litière constituée de la paille et frais d'électricité) sont plus faibles et ne représentent que $6 \%$ des charges variables. Cependant, l'alimentation qui est destinée à l'engraissement des animaux comprenne les dépenses en concentrés qui sont produits au sein de l'exploitation et la valeur des aliments achetés de l'extérieur. Les charges d'alimentation représentent $70 \%$ des charges globales de production. Ces dépenses sont en moyenne de 162101 DA par tête, variant entre 153485 DA pour les exploitations du groupe A et 170718 DA pour celles de la classe B.

\section{LES CHARGES DE STRUCTURE}

Les charges de structure comprennent les charges de main d'œuvre et de matériel. Ces dépenses représentent environ $30 \%$ du coût total moyen de production. La répartition relative des charges de structure par classe d'élevage dans le total du coût de production a tendance à augmenter en sens inverse par rapport à la taille de l'exploitation. En effet, ces charges varient d'un minimum de $130,19 \mathrm{DA} / \mathrm{kg}$ pour les exploitations du groupe $\mathrm{A}$ (exploitation de petite taille) à un maximum de $156,89 \mathrm{DA} / \mathrm{kg}$ pour celles du groupe C (exploitation de grande taille caractérisée par une diversification des activités influencée par les charges de travail supplémentaires inhérentes aux travaux culturaux). La moyenne entre les groupes d'exploitation est de l'ordre de 144,58 DA/kg (figure 5).

\section{ANALYSE DES PRODUITS}

L'examen de la structure des produits par classe d'élevage, représenté dans le figure 6, montre que la recette unitaire de vente d'un bovin varie de 600,70 DA/ $\mathrm{kg}$ vif pour les exploitations de la classe A à 601,17 $\mathrm{DA} / \mathrm{kg}$ vif pour celles du groupe $\mathrm{B}$. La recette moyenne unitaire de vente est de l'ordre de 600,93 A/kg (figure 6).

\section{ANALYSE DU PRIX DE REVIENT DE L'ACTIVITÉ VIANDE BOVINE}

L'analyse globale montre que le prix de revient moyen de l'ensemble des classes d'élevage retenues est de l'ordre de 533,64 DA/kg vif. En effet, les exploitations de la classe A affiche le prix de revient le plus faible qui est de l'ordre de $465 \mathrm{DA} / \mathrm{kg}$, suivi par les exploitations de la classe B avec $563 \mathrm{DA} / \mathrm{kg}$, puis les exploitations de classe $C$ avec un prix de revient de 1'ordre de 572,92 DA/kg (figure 7).

\section{AnalYse de la marge bRUTE de l'ACTIVITÉ VIANDE BoVINE}

La marge brute de l'activité de viande bovine correspond à la différence entre les produits et les charges opérationnelles de l'activité. La moyenne de la marge brute au cours de l'année 2011 par tête bovine est de 78 241,16 DA/tête, soit 156,48 DA/kg vif. Elle varie d'un minimum de $60706 \mathrm{DA} /$ tête, soit 121,41 DA/kg pour les exploitations appartenant à la classe $\mathrm{C}$ et d'un maximum de 91486 DA/tête, soit 182,97 DA/kg pour 
les exploitations du groupe B. En effet, le taux de la marge par rapport au coût de revient réalisé par éleveur représente $26,4 \%$. Cette différence s'explique non seulement par l'écart qui existe entre les exploitations en termes de productivité de viande bovine (rendement), mais aussi elle est imputable à l'importance des charges de l'alimentation achetée de l'extérieur (figure 8).

\section{LE PROFIT ET LE TAUX D'EFFICACITÉ ÉCONOMIQUE}

Le profit de l'éleveur est la différence entre la marge brute de l'activité et les charges de structure. Elle est en moyenne de - 13,59 DA/kg vif de viande.

\section{DISCUSSION}

\section{Prédominance des Charges d'alimentation}

Le poste dominant dans les exploitations de la région du grand Cheliff et qui représente lui seul $70 \%$ des coûts totaux est celui des charges alimentaires. En effet, les aliments achetés et qui sont destinés à l'engraissement représentent environ $90 \%$ du total de ce poste, contre $10 \%$ des charges d'aliments produits sur les exploitations.

La maîtrise du coût de production passe par l'effet cumulé d'un ensemble de facteurs de production tel que la mécanisation et l'autonomie alimentaire. Cependant, les postes d'importance intermédiaire représentent chacun $26 \%$ du coût total et son constitués par la main d'œuvre et les charges du matériel. En conséquence, l'ensemble des postes qui concourent à l'alimentation des animaux, la main d'œuvre, constitue $83 \%$ du coût total.

La politique d'élevage bovin de viande en Algérie devrait tenir compte d'une aide pour pouvoir prétendre à une diminution des coûts d'alimentation qui constituent $70 \%$ des charges de notre cheptel afin d'assurer une autonomie alimentaires des exploitations algériennes. Ainsi, pour permettre une meilleure efficacité économique de nos exploitations de bovins viande, il faut assurer un équilibre charge/produit généré par le système qui permet de dégager plus ou moins de revenu.

\section{Faible degré de mécanisation des EXPLOITATIONS}

Seulement 29 exploitations, soit $28 \%$ de l'échantillon possèdent des tracteurs, alors que le reste, soit $70 \%$ font recours à la location, ce qui augmente les frais de l'exploitation, ceci est due à la faible évolution des techniques culturales des exploitations (Sadoud, 2005). En effet, le poste charge de mécanisation et de main d'œuvre représente $30 \%$ du total des charges, on retrouve en partie les problèmes de structures agraires qui sont telles qu'il n'est pas envisageable de doter la plupart des exploitations de matériels nécessaires pour les travaux du sol.

\section{Place marginale des Cultures fourragères}

L'objectif final de la production fourragère est de mettre à la disposition du troupeau bovin viande, au meilleur coût, les fourrages de haute valeur nutritive nécessaires à ses besoins en quantité et qualité. Ce- pendant, la superficie réservée aux cultures fourragères en irrigué, occupe des taux faibles. Cette faiblesse des superficies réservées aux cultures fourragères est totalement en contradiction avec la vocation de ces exploitations de bovin viande. Les cultures fourragères occupent en moyenne environs $45 \%$ de la SAU de l'ensemble de l'échantillon. Ainsi, 50\% des éleveurs ont une superficie comprise entre 50 et $100 \%$ de la SAU et presque la moitié d'entre eux ont une superficie comprise entre 25 et $50 \%$. Par contre, $11 \%$ des éleveurs consacrent 0 à $25 \%$ de SAU pour la production fourragère. On assiste à un faible degré d'autonomie des exploitations en matière d'alimentation du cheptel qui constitue le poste le plus contraignant pour la production de viande bovine (Ferrah, 2000). L'insuffisance des ressources est commune à la plupart des éleveurs (Sadoud, 2003).

En effet, plusieurs obstacles continuent à s'opposer à l'extension des cultures fourragères dans la région, notamment:

- La priorité accordée aux cultures rémunératrices (maraîchage, arboriculture) et aux cultures vivrières (blé dur et orge principalement).

- L'absence de maîtrise de leurs itinéraires techniques ou encore l'ignorance totale des conditions de leur utilisation et de leur stockage, notamment pour l'ensilage.

- Le manque d'eau et la concurrence dans l'affectation de cette ressource rare entre les différentes spéculations (Djermoun, 2011).

\section{LA PRÉDOMINANCE DE LA LOGIQUE EXTENSIVE AU SEIN DES EXPLOITATIONS}

On peut distinguer globalement deux modes de conduite de l'élevage: le système semi intensif et le système extensif:

Le système semi intensif, est pratiqué par des éleveurs professionnels de longue date. Les conditions sont généralement favorables: étables construites en dur, puits disponibles, superficie importante, taille du troupeau varie de 50 à 100 têtes pour les exploitations. L'ensemble des exploitations, pratique les cultures fourragères, mais partiellement, car elles consacrent une grande partie de leurs superficies aux cultures les plus rentables tel que les activités maraîchères et arboricoles. Ce sont des exploitations qui pratiquent l'engraissement des jeunes, qui varient de 50 à 100 têtes.

Le système extensif ou traditionnel, pratiqué par des exploitations familiales, qui ne disposent que de superficies réduites, font recours généralement à la location des terres; ne consacrent que de petites superficies aux cultures fourragères, le reste est destiné surtout aux cultures spéculatives. Le cheptel est de taille qui varie de 10 à 20 têtes vaches laitières, constitué de races locales et améliorées. Ils recourent à l'achat de l'aliment de l'extérieur (paille, foin, son, etc.).

Ce sont des exploitations, ou leurs propriétaires exercent des activités en dehors de l'élevage, on assiste parfois à la vente de tout le cheptel par le propriétaire, suite aux problèmes de la cherté des aliments et taux de mortalité important due aux négligences sanitaires. 


\section{ContrainTES TECHNIQUES LIÉES AUX SYSTÈmes d'ÉLEVAGE E DE PRODUCTION DE VIANDE BOVINE}

Parmi les contraintes de la faiblesse de la production de viande bovine, c'est la dominance du système d'élevage à caractère familial et traditionnel, avec des troupeaux de faibles tailles, ce qui entrave l'introduction de l'innovation technique. Ainsi, la pratique d'un élevage de type familial (86\%), constitue une contrainte de l'intensification de la production de viande bovine. La faiblesse de technicité chez les éleveurs dans la maîtrise de la conduite du troupeau et de rationnement de l'alimentation, de l'utilisation des techniques de reproduction, gène la réalisation du progrès (Kherzat, 2007).

\section{CONCLUSION}

L'analyse des prix de revient à la production par type d'élevage montre clairement des différences de grandeurs importantes entre les types d'élevage de la région d'étude, du groupe le plus rentable et le plus performant aux groupes les moins performants. En effet, de l'étude sur l'évaluation des performances économiques des exploitations de bovin viande, il ressort que les exploitations de (Classe A) peuvent être considérées comme le groupe le plus performant de l'ensemble des types distingués avec un coût de production parmi les plus bas. Par contre les autres groupes (Classe B et C) paraissent dans des conditions difficiles suite aux difficultés et contraintes des facteurs de production locaux (foncier, fourrages) ou importés (aliments concentrés, fourrages) qui suivraient certainement le mouvement ascendant des prix. Ces exploitants seraient des victimes de la volatilité des prix. La condition de leur survie serait la chute des prix des aliments de bétail.

Le développement des exploitations d'élevage bovin à viande de la région du grand Cheliff, se trouve handicapé par le manque d'organisation des producteurs de viande bovine. En plus du soutien de la production de viande bovine en Algérie qui reste très faible.

Les exploitations d'élevage bovin à viande de la région ne pourraient avoir comme alternative qu'une stratégie défensive pour assurer leurs survies très vulnérables aux aléas externes.

\section{BIBLIOGRAPHIE}

Abbas, K.; Madani, T. et Abdelgherfi, A. 2011 . Le secteur des productions animales en Algérie. Une approche territoriale pour un développement durable. 6è Journées de Recherches sur les Productions Animales. Université Mouloud Mammeri. Algérie. 110 pp.

Ali-Benamara, B. 1985. Analyse de fonctionnement du système de spécialisation dans l'élevage. Exemple de l'engraissement des taurillons dans le périmètre irrigué d'El-khemis. Thèse Ingénieur Agronome. INA. Alger. $85 \mathrm{pp}$.

Berchiche, A. 2003. Les productions animales en Algérie. 4 tournées de Recherche sur les Productions Animales. Université Mouloud Mammeri. Algérie. 160 pp.

Chambr' Agri. 2011. Les coûts de production dans les élevages bovins viande. Réseaux d'élevage pour le conseil et la prospective. Collection THEMA. France.

Diermoun, A. 2011. Effet de l'adhésion de l'Algérie à l'OMC et à la zone de libre échange Union Européenne/Pays tiers Méditerranéens sur la filière lait. Thèse Doctorat en Développement rural. ENSA. Alger. Algérie. 478 pp.

Ferrah, A. 2000. L'élevage bovin laitier en Algérie: Problématique, questions et hypothèses pour la recherche. $3^{3}$ Journées de Recherches en Productions Animales, Conduite et Performance d'Élevage. Université Mouloud Mammeri. Algérie. pp. 40-49.

Institut de l'élevage. 2011 . Performance économique des élevages bovins viande. Les bons repères pour prendre les décisions. Collection THEMA. 7 pp.

Kherzat, B. 2007. Essai d'évaluation de la politique laitière en perspective de l'adhésion de l'Algérie à l'OMC et à la zone de libre-échange avec I'Union Européenne. Mémoire de Magister, Institut National Agronomique d'Alger. Economie Rurale. Alger. Algérie. 120 pp.

Landais, E. 1987. Recherches sur les systèmes d'élevage: questions ef perspectives. INRA Versailles, France. (Document de travail). 75 pp.

Pons, V. et Pouch, T. 2013. Viande bovine: une décennie de mutations sur le marché mondial. Chambres d'Agriculture, 1021: 7-10.

Raimbault, G. 2004. Comptabilité générale et analyse financière. Chihab Eyrolles. Alger. 122 pp.

Sadoud, M. 2011. Place de l'activité bouchère dans la filière viande rouge algérienne. Arch Zootec, 60: 309-312.

Sadoud M. 2005. Mécanisation agricole en Algérie. Congrès International de la Mécanisation Agricole. L'Efficacité de la Mécanisation Agricole et son Impact Environnemental, 9-11 décembre. Tunisie. 430 pp.

Sadoud, M. 2003. Le marché du bétail et de la viande rouge dans la wilaya de Chlef. 4è Journées de Recherches sur les Productions Animales. Université de Tizi Ouzou. Algérie. pp. 43-51.

Soltner D. 1981. La production de viande bovine. Collection science et technique agricole. 13è ed. Angers. Sainte Gemmes sur Loire, France. 83 pp.

UBIFRANCE. 2014. Le marché de la filière viande en Algérie. Business France. $50 \mathrm{pp}$. 
